\title{
Lecture 12- Entanglement
}

\author{
Fred Jendrzejewski ${ }^{1}$, Selim Jochim ${ }^{2}$, and Matthias Weidemüller ${ }^{2}$ \\ ${ }^{1}$ Kirchhoff-Institut für Physik \\ ${ }^{2}$ Physikalisches Institut der Universität Heidelberg
}

April 12, 2021

\begin{abstract}
We will discuss the creation of entangled photons and how they can be used for the test of Bell's inequalities.

We have previously discussed how complicated gain media allow for the amplification light (Jendrzejewski et al.). Here we will discuss how it is used to create entangled photons and then dive into the fundamental test of the Bell inequalities.
\end{abstract}

\section{Parametric down-conversion}

Fig. 1 shows the schematic setup of an experiment where pairs of entangled photons are created by a two-photon source. Two polarizers can be used to probe the polarization of the photons.

\section{$1.1 \quad$ Three-wave mixing}

The crystal in the medium is a non-linear crystal, which means that we can write the polarization is not just linear, but higher order terms will play a role. We will consider for starters that there are actually two pump waves in the same direction, which allows us to write:

$$
P_{N L}(z)=2 \epsilon_{0} \chi^{(2)} \mathcal{E}_{1}(z) \mathcal{E}_{2}(z) e^{i\left(k_{1}+k_{2}\right) z}
$$

This non-linear polarizability leads to the following equations of motion (gry):

$$
\begin{aligned}
\frac{d \mathcal{E}_{3}}{d z} e^{i k_{3} z} & =\frac{i \omega_{3}}{2 \epsilon_{0} n_{3} c} P_{N L}(z) \\
\frac{d \mathcal{E}_{3}}{d z} & =\frac{i \omega_{3}}{n_{3} c} \chi^{(2)} \mathcal{E}_{1}(z) \mathcal{E}_{2}(z) e^{i\left(k_{1}+k_{2}-k_{3}\right) z}
\end{aligned}
$$

where $\omega_{3}=\omega_{1}+\omega_{2}$. We can now additionally assume that:

- The effect of the medium does not change the strong pump to much.

- The amplified field is zero initially.

- The oscillating phase factor $\left(k_{1}+k_{2}-k_{3}\right) z$ can be ignored, i.e. where we have:

$$
\vec{k}_{3}=\vec{k}_{1}+\vec{k}_{2}
$$


We can then simplify to:

$$
\frac{d \mathcal{E}_{3}}{d z}=\frac{i \omega_{3} \chi^{(2)}}{n_{3} c} \mathcal{E}_{1} \mathcal{E}_{2}
$$

So the amplitude of the mixed field increases in a linear fashion in the non-linear medium. However, the typical amplitude for production is below $1 \%$ for commonly used crystals.

\section{Polarization entangled photons}

We will try to observe correlations between the photons. Two optical fibers are collecting the pairs of photons and transmit them to the single photon detectors. Finite collection and detection efficiency causes only one of the two photons to be detected in most cases. Therefore, a coincidence circuit registers events in which two photons arrive within $30 \mathrm{~ns}$. As the rate of detected individual photons is about $50 \mathrm{kHz}$, we assume that photons arriving during such a small time window were created in the same event.

a)

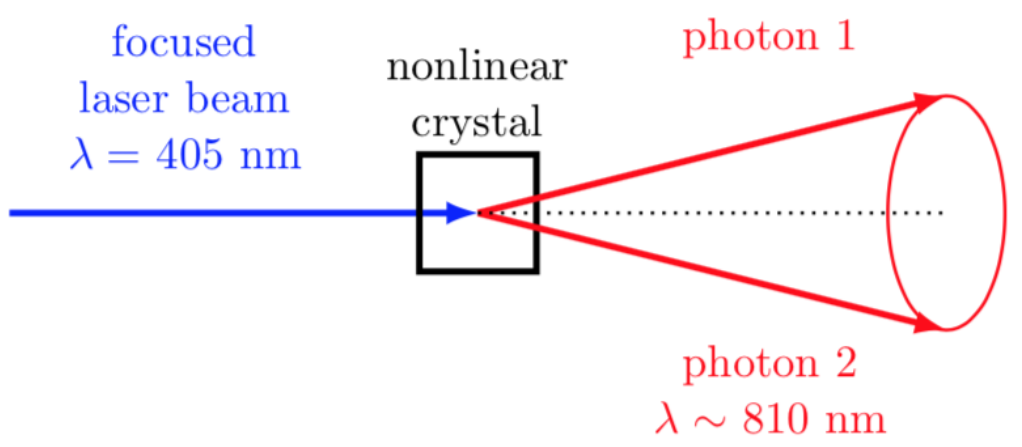

b)

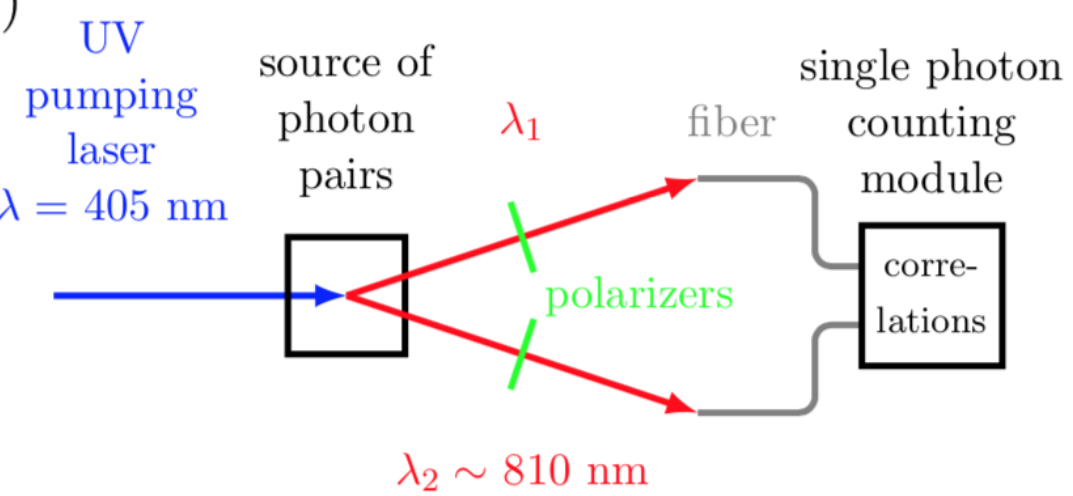

Figure 1: a) The process of spontaneous parametric down-conversion (SPDC). Inside a nonlinear crystal, two outgoing photons are created from an incoming photon. Momentum and energy conservation apply, resulting in a characteristic emission cone. b) Two photons created by SPDC encounter polarizers. Depending on the polarization, the photons are either absorbed or transmitted. After passing the polarizers, the photons are collected with two optical fibers and detected with single photon counters. The detector indicates a coincidence when both photons arrive within roughly 30 ns. 


\subsection{Polarization analysis}

To study the quantum nature of the correlations, we will employ polarizers and later dive into Bell's inequalities as well as entanglement in general. The interested reader will have a great time reading through the complement 5.C of (gry).

The first emitted photon is analyzed by a rotatable polarizers $\hat{A}(\theta)$, which has two detection paths \pm 1 . The other polarizer will be called $\hat{B}(\theta)$ the only difference is that he only acts on photon 2 . Basically, we are following the Alice and Bob notation here.

We can express it then in our basis states of vertical polarization $|V\rangle$ and horizontal polarization $|H\rangle$. The polarizer aligned with $H$ has eigenvalues:

$$
\begin{aligned}
\hat{A}|H\rangle & =+|H\rangle \\
\hat{A}|V\rangle & =-|V\rangle
\end{aligned}
$$

To analyse the polarization of each photon in detail we can also rotate the polarizer by an angle of $\theta$. In this case the transmitted eigenstates are:

$$
\begin{aligned}
& |\theta\rangle_{+}=\cos (\theta)|H\rangle+\sin (\theta)|V\rangle \\
& |\theta\rangle_{-}=-\sin (\theta)|H\rangle+\cos (\theta)|V\rangle
\end{aligned}
$$

Taking as input states $|H\rangle$, we simply find Malus law:

$$
\begin{aligned}
& P_{+}(\theta)=\cos (\theta)^{2} \\
& P_{-}(\theta)=\sin (\theta)^{2}
\end{aligned}
$$

In the rotated basis we can express the polarization operator as:

$$
\hat{A}(\theta)=\left(\begin{array}{cc}
\cos (2 \theta) & \sin (2 \theta) \\
\sin (2 \theta) & -\cos (2 \theta)
\end{array}\right)
$$

We can now employ the two polarizers to investigate the two emitted photons as shown in Fig. 2.

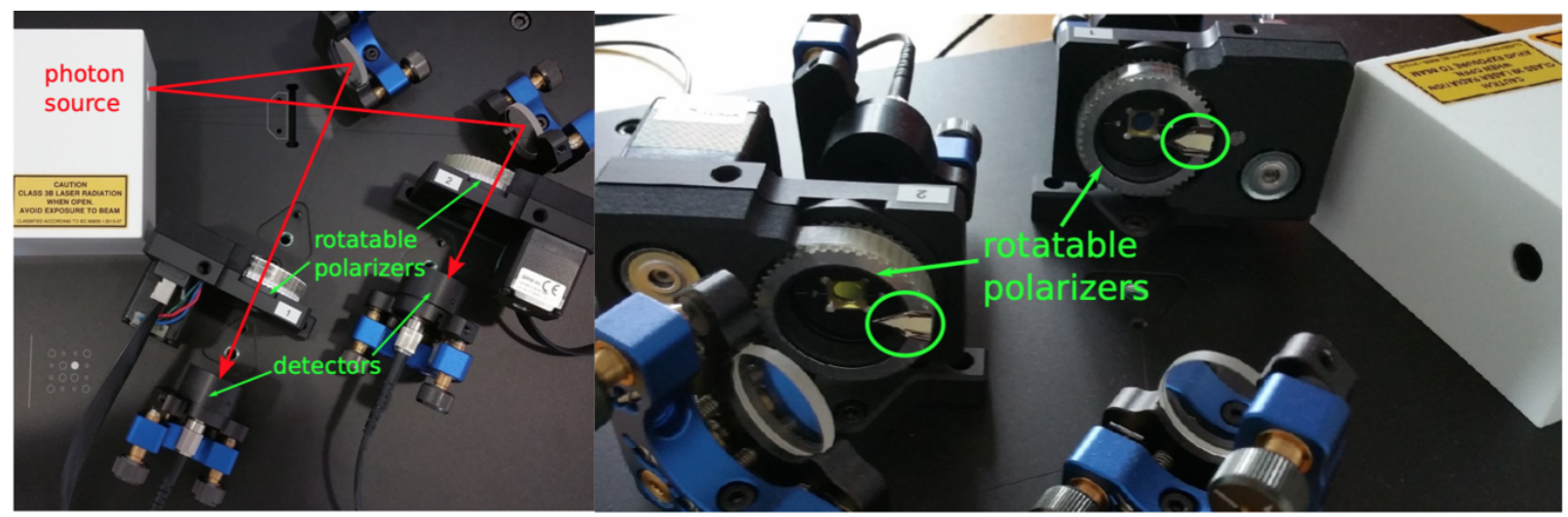

Figure 2: Polarization analysis of correlated photons

The possible outcome of our experiments are the four states $\{H H, H V, V H, V V\}$ and hence we could decompose our full wavefunction as:

$$
|\psi\rangle=c_{0}|H H\rangle+c_{1}|H V\rangle+c_{2}|V H\rangle+c_{3}|V V\rangle
$$


Using the two polarizers we can now start to investigate the prefactors of the full wavefunction. Let us first look into the results of a polarizer that is not rotated. We find:

$$
\langle\psi|\hat{A}| \psi\rangle=\left|c_{0}\right|^{2}+\left|c_{1}\right|^{2}-\left|c_{2}\right|^{2}-\left|c_{3}\right|^{2}
$$

For Bobs polarizer in the same position we would find:

$$
\langle\psi|\hat{B}| \psi\rangle=\left|c_{0}\right|^{2}+\left|c_{2}\right|^{2}-\left|c_{1}\right|^{2}-\left|c_{3}\right|^{2}
$$

\subsection{An equivalent 2 qubit circuit}

The optics setup handles two independent photons, with two outcomes each. So we can also see the presented setup as a two qubit system. A circuit diagram would mostly look the following way.

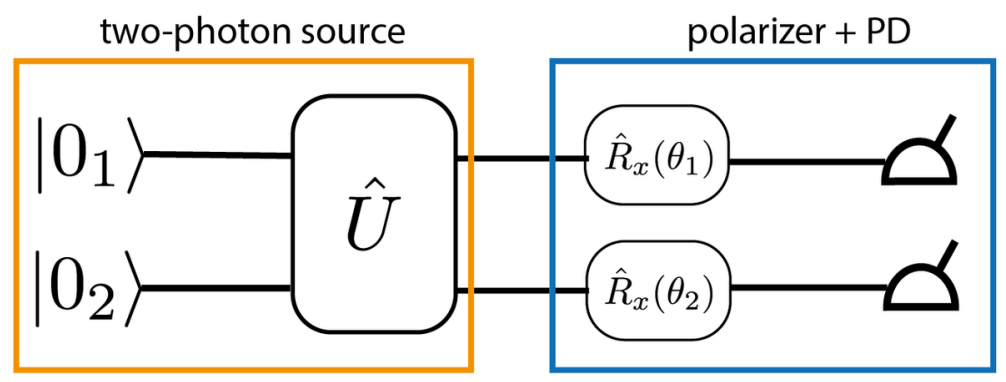

Figure 3: Realizing the two-photon experiment within a quantum circuit. What are the correlations the two photons have?

The two photons originate from an unknown source, which is here modelled by photons propagating through some unitary matrix $\hat{U}$. The measurement is performed in the last step, projecting the qubit on its up or down state. The rotation around the $\mathrm{x}$ axis $\hat{R}_{x}$ transforms a qubit state into a superposition. In strong analogy to the polarization

\section{$2.3 \quad$ A naive guess}

We know that we have two photons in the system. Both can have some polarization and clearly they are propagation in different directions. So it does not seems to much of a stretch to guess that the total wavefunction is the product of two superposition states:

$$
\begin{aligned}
|\psi\rangle_{p} & =\left(c_{H, 1}|H\rangle+c_{V, 1}|V\rangle\right) \otimes\left(c_{H, 2}|H\rangle+c_{V, 2}|V\rangle\right) \\
& =\left(c_{H, 1} c_{H, 2}|H H\rangle+c_{V, 1} c_{V, 2}|V V\rangle+c_{V, 1} c_{H, 2}|V H\rangle+c_{H, 1} c_{V, 2}|H V\rangle\right)
\end{aligned}
$$

\subsection{The experimental observation of entanglement}

- We find a lot of counts if both polarizers are set vertical or horizontal. So the state has a $V V$ and a $H H$ component, which tells us that $c_{0}$ and $c_{3}$ are non-zero. The equal rate of measuring further tells us that they are roughly similiar in amplitude, so we can write for simplicity $\left|c_{0}\right|=\left|c_{3}\right|$

- We find zero correlation if the polarizers are opposite. So the mixed terms are zero $c_{1}=c_{2}=0$ 
In summary we can expect the state to be written as:

$$
\left|\psi_{B}\right\rangle=\frac{|H H\rangle+|V V\rangle}{\sqrt{2}}
$$

This is quite clearly incompatible with our naive guess (18), which means that we have an entangled state.

\section{Optional: Quantifying entanglement}

We will study the properties of the entangled states later in more detail. However, we will take a short moment to cite two ways of quanitfying the entanglement through the density operator:

$$
\hat{\rho}=\sum_{i} \eta_{i}|i\rangle\langle i|
$$

- The reduced density operator, which shows mixed states if there is entanglement:

$$
\hat{\rho}_{1}=\operatorname{tr}_{2}(\hat{\rho})
$$

In this case, $\hat{\rho}$ is the density operator of a pure state and $\operatorname{tr}_{2}$ is the trace over the Hilbert space of particle 2 .

- The von Neumann entropy, which measures the remaining uncertainty within a quantum state:

$$
\begin{aligned}
S & =-\operatorname{tr}(\hat{\rho} \ln \hat{\rho}) \\
& =-\sum_{i} \eta_{i} \ln \eta_{i}=\sum_{i} \eta_{i} \ln \frac{1}{\eta_{i}}
\end{aligned}
$$

For the Bell states of (20) we find then:

$$
\hat{\rho_{B 1}}=\frac{1}{2}(|H\rangle\langle H|+| V\rangle\langle V|)
$$

Its corresponding entropy is $S=\ln 2$, the entropy of a pure state is $S=0$.

\section{Back to the correlation between distant photons}

In the last sections we performed measurements on joined detection probabilities between two independent polarizers. Quite importantly we saw that:

- Each photon is in a superposition of $|H\rangle$ and $|V\rangle$.

- Both photons are always detected in the same polarization state.

- From Fig. 2 it seems as if 1 was a bit closer to the source than $2^{1}$. So 1 is detected a bit earlier and projected onto one of the two states.

- Yet, 2 seems to instantaneously on which polarization 1 was projected and choses the same one.

For our set-up the distances are small, but the same observations and arguments hold also for very large distances between the detectors. Einstein, Podolski and Rosen understood this long distance correlation and decided that something was funky about quantum mechanics (Einstein et al., 1935).

\footnotetext{
${ }^{1}$ The exact order does not matter, but they are most certainly not at exactly the same distance from the source
} 
Therefore, the idea of an additional hidden shared parameter can be introduced to explain the correlations between distant objects. We will simply assume that the two photons have well-defined polarization with angle $\lambda$ from the starting point, yet this polarization varies randomly from pair to pair between 0 and $2 \pi$. Hence we have uniform probability distribution $\rho(\lambda)=\frac{1}{2 \pi}$. The measurement of the polarizers can then simply be modelled through

$$
A_{h v}(\lambda, \theta)=\operatorname{sign}(\cos 2(\theta-\lambda))
$$

This model reproduces nicely all the tests that we ran previously. Namely, maximum detection for HH and $\mathrm{VV}$ as well as zero correlation for $H V$. They can be nicely compared through the correlation coefficient $E\left(\theta_{1}, \theta_{2}\right)$. The particularly perturbing case is that this simple model for hidden parameters works even perfectly well in the case of $45^{\circ}$ angles. So is there any measurable difference between our observations and the hidden variable models?

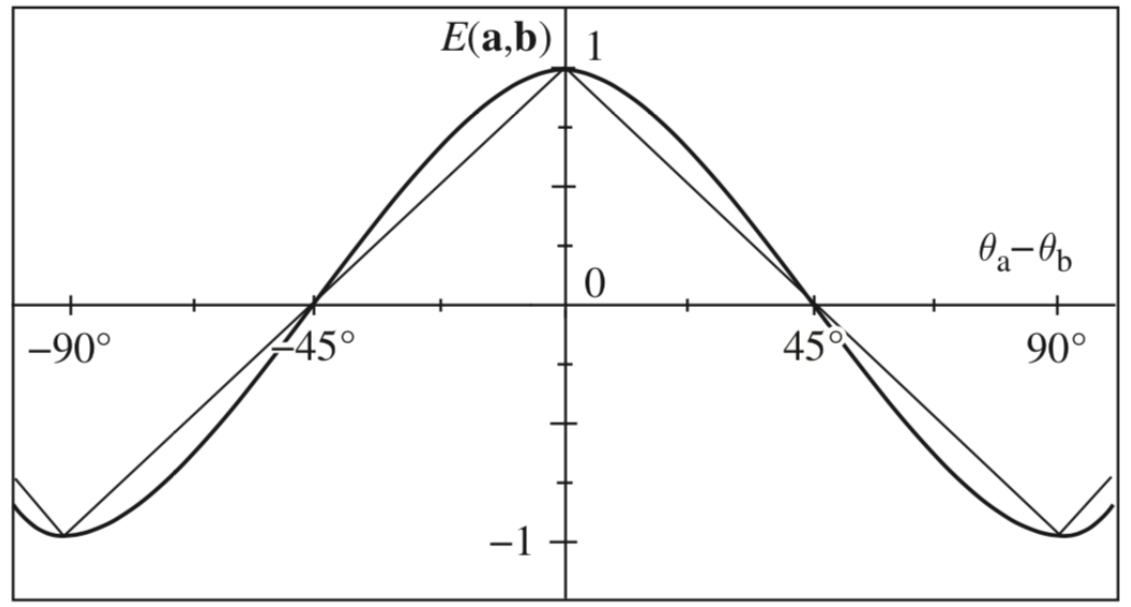

Figure 4: Correlation coefficient as taken from (gry)

\section{Bell's theorem}

Bell posed the previous discussion on a more general and quite simple footing (Bell, 1964) and later extended by Clauser, Horner, Shimony, Holt (Clauser et al., 1969). For the hidden parameter we need just a standard density distribution with:

$$
\begin{aligned}
\rho(\lambda) & \geq 0 \\
\int d \lambda \rho(\lambda) & =1
\end{aligned}
$$

We additionally should describe the polarizer by some function that takes the value \pm 1 depending on the angle of the polarizer and the hidden variable:

$$
\left|A\left(\lambda, \theta_{1}\right)\right|=\left|A\left(\lambda, \theta_{2}\right)\right|=1
$$

In the experiment we now have two polarizers $A$ for Alice and $B$ for Bob, which we will allow to be in some position $\theta$ as visualized in Fig. 5 . 


$$
\begin{array}{|lcc}
\text { Alice } & \frac{|H H\rangle+|V V\rangle}{\sqrt{2}} & \text { Bob } \\
\hline A\left(\theta_{1}\right)= \pm 1 & \longleftrightarrow \begin{array}{l}
B\left(\theta_{2}\right)= \pm 1 \\
B\left(\theta_{2}^{\prime}\right)= \pm 1
\end{array}
\end{array}
$$

Figure 5: A Bell experiment

We will now calculate the the expectation value for joint detection:

$$
E\left(\theta_{1}, \theta_{2}\right)=\overline{A\left(\theta_{1}\right) B\left(\theta_{2}\right)}-\overline{A\left(\theta_{1}\right)} \overline{B\left(\theta_{2}\right)}
$$

We can simplify further for equal probability of having $\mathrm{H}$ or $\mathrm{V}$ polarization, which leads too:

$$
\begin{aligned}
E\left(\theta_{1}, \theta_{2}\right) & =\overline{A\left(\theta_{1}\right) B\left(\theta_{2}\right)} \\
& =\int d \lambda A\left(\lambda, \theta_{1}\right) B\left(\lambda, \theta_{2}\right)
\end{aligned}
$$

Bells inequalities are then studying the correlations between photons in four different configuations:

$$
\begin{aligned}
s\left(\lambda, \theta_{1}, \theta_{1}^{\prime}, \theta_{2}, \theta_{2}^{\prime}\right) & =A\left(\lambda, \theta_{1}\right) B\left(\lambda, \theta_{2}\right)-A\left(\lambda, \theta_{1}\right) B\left(\lambda, \theta_{2}^{\prime}\right)+A\left(\lambda, \theta_{1}^{\prime}\right) B\left(\lambda, \theta_{2}\right)+A\left(\lambda, \theta_{1}^{\prime}\right) B\left(\lambda, \theta_{2}^{\prime}\right) \\
& =A\left(\lambda, \theta_{1}\right)\left(B\left(\lambda, \theta_{2}\right)-B\left(\lambda, \theta_{2}^{\prime}\right)\right)+A\left(\lambda, \theta_{1}^{\prime}\right)\left(B\left(\lambda, \theta_{2}\right)+B\left(\lambda, \theta_{2}^{\prime}\right)\right) \\
& = \pm 2
\end{aligned}
$$

We actually have no access to the hidden parameter, so we are looking for its average value:

$$
\begin{array}{r}
S=\int d \lambda \rho(\lambda) s\left(\lambda, \theta_{1}, \theta_{1}^{\prime}, \theta_{2}, \theta_{2}^{\prime}\right) \\
-2 \leq S \leq 2
\end{array}
$$

And this value can now be measured experimentally as we can identify:

$$
S=E\left(\theta_{1}, \theta_{2}\right)-E\left(\theta_{1}, \theta_{2}^{\prime}\right)+E\left(\theta_{1}^{\prime}, \theta_{2}\right)+E\left(\theta_{1}^{\prime}, \theta_{2}^{\prime}\right)
$$

This is known as the Bell-Clauser-Horn-Shimony-Holt (CHSH) inequalities.

\subsection{The inconsistency between hidden parameters and quantum mechanics}

We can now go again through the predictions of quantum mechanics and test if there is a region of interest in which we should observe a violation of the CHSH inequalities. Actually there is an important configuration at which we should break them rather violantly namely for:

$$
\begin{aligned}
\left|\theta_{1}-\theta_{2}\right| & =\frac{\pi}{8}\left(22.5^{\circ}\right) \\
\left|\theta_{1}^{\prime}-\theta_{2}\right| & =\frac{\pi}{8}\left(22.5^{\circ}\right) \\
\left|\theta_{1}^{\prime}-\theta_{2}^{\prime}\right| & =\frac{\pi}{8}\left(22.5^{\circ}\right) \\
\left|\theta_{1}-\theta_{2}^{\prime}\right| & =\frac{3 \pi}{8}\left(67.5^{\circ}\right)
\end{aligned}
$$

Here, we expect to have $S=2 \sqrt{2}$. So to test Bells inequalities we have to measure the joint probabilities in rather unusual angles. This also explains why quantum mechanics and local hidden variables seem so similiar in this kind of experiments, the biggest differences are hard to see accidentally. 


\subsection{The experimental test}

We can now study the correlations for the following configuration.

$$
\begin{gathered}
\theta_{1}=0 \text { and } \theta_{1}^{\prime}=\frac{\pi}{4} \\
\theta_{2}=\frac{\pi}{8} \text { and } \theta_{2}^{\prime}=\frac{3 \pi}{8}
\end{gathered}
$$

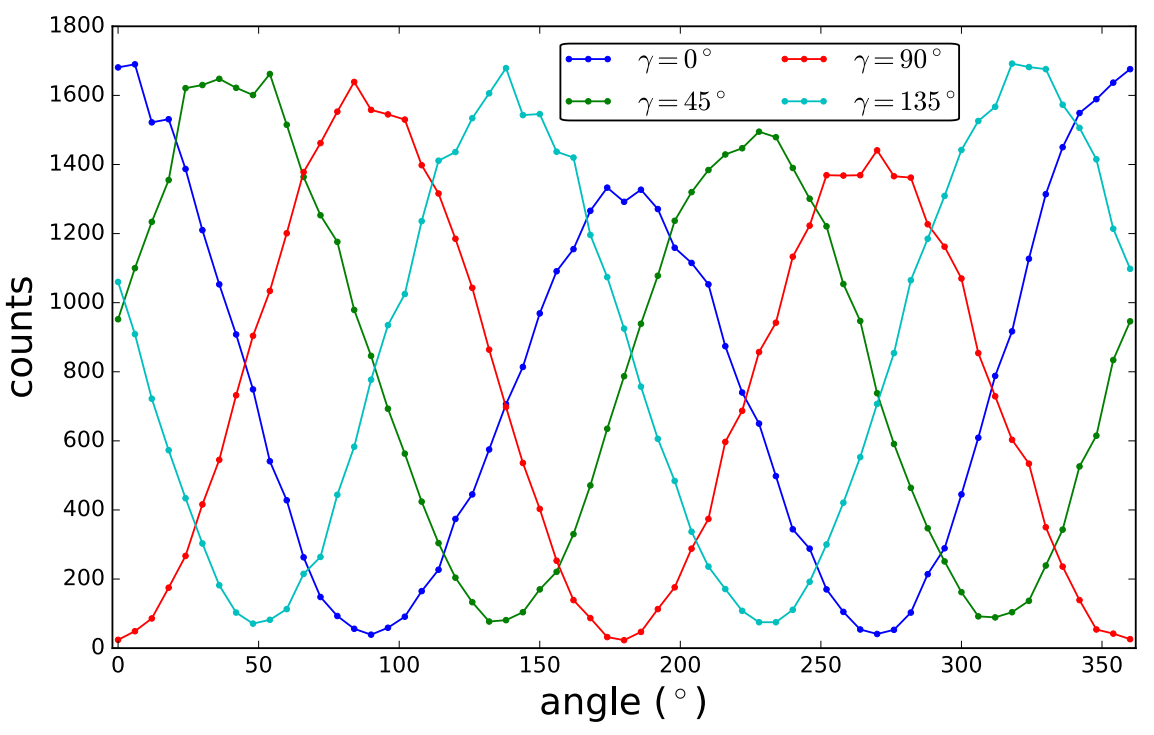

Figure 6: Correlation measurement between the two photons measured with the setup shown in $1 \mathrm{~b}$ ) and 2 . One of the rotatable polarizers stays at an angle $\gamma \in\left\{0^{\circ}, 45^{\circ}, 90^{\circ}, 135^{\circ}\right\}$ while the other polarizer is rotated counter-clockwise in small steps between $0^{\circ}$ and $360^{\circ}$.

Experimentally we observe quite frequently value above 2. However, please be aware that there are a lot of loopholes in our test. The most obvious ones are:

1. Position of the polarizers is not random.

2. The detectors are not well separated.

Other loopholes exist, but all realistically known loopholes have been closed over the course of the last three decades. (Giustina et al., 2015; Shalm et al., 2015; Hensen et al., 2015).

\section{References}

Introduction to Quantum Optics by Gilbert Grynberg. https://www.cambridge.org/core/books/introductionto-quantum-optics/F45DCE785DC8226D4156EC15CAD5FA9A. URL / core/books/introduction-toquantum-optics/F45DCE785DC8226D4156EC15CAD5FA9A. Accessed on Fri, November 23, 2018.

J. S. Bell. On the Einstein Podolsky Rosen paradox. Physics Physique, 1(3):195-200, nov 1964. doi: 10. 1103/physicsphysiquefizika.1.195. URL https://doi.org/10.1103\%2Fphysicsphysiquefizika.1.195. 
John F. Clauser, Michael A. Horne, Abner Shimony, and Richard A. Holt. Proposed Experiment to Test Local Hidden-Variable Theories. Physical Review Letters, 23(15):880-884, oct 1969. doi: 10.1103/physrevlett. 23.880. URL https://doi.org/10.1103\%2Fphysrevlett.23.880.

A. Einstein, B. Podolsky, and N. Rosen. Can Quantum-Mechanical Description of Physical Reality Be Considered Complete? Physical Review, 47(10):777-780, may 1935. doi: 10.1103/physrev.47.777. URL https://doi.org/10.1103\%2Fphysrev. 47.777.

Marissa Giustina, Marijn A. M. Versteegh, Sören Wengerowsky, Johannes Handsteiner, Armin Hochrainer, Kevin Phelan, Fabian Steinlechner, Johannes Kofler, Jan-Åke Larsson, Carlos Abellán, Waldimar Amaya, Valerio Pruneri, Morgan W. Mitchell, Jörn Beyer, Thomas Gerrits, Adriana E. Lita, Lynden K. Shalm, Sae Woo Nam, Thomas Scheidl, Rupert Ursin, Bernhard Wittmann, and Anton Zeilinger. SignificantLoophole-Free Test of Bell's Theorem with Entangled Photons. Physical Review Letters, 115(25), dec 2015. doi: 10.1103/physrevlett.115.250401. URL https://doi.org/10.1103\%2Fphysrevlett.115.250401.

B. Hensen, H. Bernien, A. E. Dréau, A. Reiserer, N. Kalb, M. S. Blok, J. Ruitenberg, R. F. L. Vermeulen, R. N. Schouten, C. Abellán, W. Amaya, V. Pruneri, M. W. Mitchell, M. Markham, D. J. Twitchen, D. Elkouss, S. Wehner, T. H. Taminiau, and R. Hanson. Loophole-free Bell inequality violation using electron spins separated by 1.3 kilometres. Nature, 526(7575):682-686, oct 2015. doi: 10.1038/nature15759. URL https://doi.org/10.1038\%2Fnature15759.

Fred Jendrzejewski, Selim Jochim, and Matthias Weidem ller. Lecture 10 - Propagation of light in dielectric media. URL https://doi.org/10.22541\%2Fau.154221124.43729319.

Lynden K. Shalm, Evan Meyer-Scott, Bradley G. Christensen, Peter Bierhorst, Michael A. Wayne, Martin J. Stevens, Thomas Gerrits, Scott Glancy, Deny R. Hamel, Michael S. Allman, Kevin J. Coakley, Shellee D. Dyer, Carson Hodge, Adriana E. Lita, Varun B. Verma, Camilla Lambrocco, Edward Tortorici, Alan L. Migdall, Yanbao Zhang, Daniel R. Kumor, William H. Farr, Francesco Marsili, Matthew D. Shaw, Jeffrey A. Stern, Carlos Abellán, Waldimar Amaya, Valerio Pruneri, Thomas Jennewein, Morgan W. Mitchell, Paul G. Kwiat, Joshua C. Bienfang, Richard P. Mirin, Emanuel Knill, and Sae Woo Nam. Strong Loophole-Free Test of Local Realism. Physical Review Letters, 115(25), dec 2015. doi: 10.1103/physrevlett.115.250402. URL https://doi.org/10.1103\%2Fphysrevlett.115. 250402. 